\title{
Editorial
}

\section{COMPLICATIONS OF TREATMENT WITH CLOFAZIMINE (LAMPRENE: B663)}

Much has been written on the subject of this dye since Browne and Hogerzeil (1962) first reported its good effect in the treatment of leprosy, and, as regards its side effects, most are by now well known. These include red-brown pigmentation of skin and conjunctiva with darkening of skin lesions; red colouration of urine, stools, sputum and sweat; dryness of skin, particularly of forearms and lower legs, which may progress to typical ichthyosis; and, less commonly, irritation of skin lesions. The side effect of clofazimine which is less well known, and which has been highlighted in the paper by Plock and Leiker which appears in this Number of Leprosy Review, is its effect on the gastro-intestinal tract. The first reference to this came from Williams et al. (1965); of 3 patients treated with the drug, one developed diarrhoea and colicky abdominal pain, and one other patient experienced malaise, anorexia and weight loss, but no abnormalities were detected on x-ray examination. Atkinson et al. (1967) reported that a patient of theirs, after several months of treatment, developed anorexia, epigastric pain and occasional vomiting, followed by marked loss of weight. Tests for malabsorption were negative, but x-ray examination revealed coarsening of mucosal pattern and segmentation of barium in ileum and distal jejunum. A specimen of jejunum was obtained by biopsy and showed a normal mucosal villous pattern and moderate numbers of plasma cells in the lamina propria. In addition, red crystals were seen in the lamina propria and were identified by the ultra-violet spectroscope as clofazimine crystals. Pettit et al. (1967) recorded that one of their patients complained of intermittent diarrhoea and upper abdominal pain during the third and fourth months of treatment, but was able to complete the 6 months trial without interruption. Inkamp (1968) treated 18 patients with $200 \mathrm{mg}$ of clofazimine daily and noted that 3 patients developed diarrhoea; one after 3 weeks, one after 7 weeks, and the third after 11 months. Helmy et al. (1971) treated 10 patients with $300 \mathrm{mg}$ daily given as a single dose, and 2 patients experienced nausea, vomiting and epigastric pain early in the course, but their symptoms settled when the daily dose was divided. Schulz (1971) treated 123 patients with clofazimine and 3 developed abdominal symptoms consisting of pain and bowel irregularity severe enough to stop treatment. They had been treated for 12,13 and 18 weeks respectively, and the maximum daily dose was $300 \mathrm{mg}$ in 2 cases and $400 \mathrm{mg}$ in the third. Weight loss was so marked in one patient that he was admitted to hospital, but all routine investigations, including x-ray examination, were negative; jejunal biopsy was not done. He recovered soon after the drug was stopped. The other 2 patients subsequently tolerated clofazimine in reduced dosage over several months, while in 10 other patients who complained of transient abdominal pain and nausea in the early stages no 
alteration in treatment was required. In a series of 120 patients treated by Karat (1975) 2 suffered from recurrent colicky abdominal pain after 6 months and 18 months respectively. Barium meal studies showed narrowing of the terminal ileum and dilatation of proximal loop. There was no change in the absorptive functions of the small intestine. At laparotomy about 6 inches of terminal ileum appeared thickened and oedematous, and some enlarged mesenteric lymph nodes were found. Histological examination of these nodes and of terminal ileum showed a non-specific granuloma characterized by foreign body giant cells and lymphocytes, together with crystals of clofazimine. No acid-fast bacilli were grown on culture. Clofazimine was withdrawn and the symptoms cleared up in 8 to 10 weeks. Desikan et al., (1975) have reported autopsy findings in a young Indian woman who had suffered from lepromatous leprosy complicated by severe lepra reaction and nephrotic syndrome. The story was that prednisolone had failed to control the reaction so clofazimine was added to her treatment, $300 \mathrm{mg}$ daily, but had to be stopped after 4 months because of diarrhoea which had not responded to reduced dosage. She died a month later, and post-mortem examination revealed a striking colouration of all tissues within chest and abdomen, the colour varying from orange-red to brick-red. There was congestion and oedema of the mucosa of small and large intestine, more pronounced in the former, and histological examination of mucosa and submucosa showed cellular infiltration and oedema together with clofazimine crystals. Similar crystals were also present in liver, spleen and lung. Another finding was widespread amyloidosis, and the fact that the adrenal cortex was seen as a mass of amyloid material confirmed that death had been due to adrenal failure. Harman (1975) has given me details of a Burmese lady who came to England in 1967, was found to be suffering from lepromatous leprosy the following year, and was treated with dapsone. In 1969 treatment was changed to clofazimine because of continued type 2 lepra reaction (ENL reaction), and when she moved to Bristol she came under Dr Harman's care. Clofazimine was continued, dosage varying between 100 and $600 \mathrm{mg}$ daily, and between March 1972 and May 1975 she lost weight and suffered from recurring anorexia, nausea, dirrhoea and abdominal pain. Many investigations were carried out during this time, but failed to establish a diagnosis. Clofazimine was stopped in May 1975 and her gastro-intestinal symptoms improved, but she continued to lose weight (from $38 \mathrm{~kg}$ in May to $27.5 \mathrm{~kg}$ in September). On 9 September she was admitted to hospital with severe gastro-intestinal symptoms, and 5 days later she died. The cause of death was considered to be acute electrolyte imbalance. At autopsy the typical pigmentation of viscera was seen, and crystals of clofazimine were found in the lamina propria of the small bowel. Mesenteric nodes could not be identified as they were replaced by necrotic brown slime.

One of my patients has demonstrated that clofazimine crystals can be found in mesenteric lymph nodes nearly 4 years after stopping the drug. He is an adult male under treatment for lepromatous leprosy, and clofazimine therapy was instituted in March 1967 because of prolonged type 2 lepra reaction. He continued on the drug until December 1971, a total period of 4 years and 9 months, dosage varying from 100 to $200 \mathrm{mg}$ daily for the first $3 \frac{1}{4}$ years, reducing to $100 \mathrm{mg}$ every alternate day for the next 12 months, and to $100 \mathrm{mg}$ twice a week for the last 6 months. During this time all skin smears became negative, and in December 1971 treatment was changed to dapsone. In October 1974 he began to suffer from diarrhoea and epigastric pain which persisted in spite of various 
symptomatic treatments and replacing dapsone by thiambutosine. Stool examinations and x-ray investigations were negative. By October 1975 his gastro-intestinal symptoms were so distressing that a laparotomy was performed, and at operation there were no pigmentary changes in the abdominal viscera and the intestine appeared normal. Mesenteric lymph nodes were enlarged, and one was removed for histological examination together with a small piece of distal jejunum. Dr D. S. Ridley reported as follows:

"Jejunum: The histology is within normal limits except perhaps for an excess of mucus secretion. Mesenteric lymph node: This shows sinus catarrh. There are some large foamy macrophages heavily loaded with ceroid pigment which is a feature of clofazimine treatment. It is impossible to identify bacilli in the macrophages because of the pigment. Cryostat sections show dense deposits of clofazimine crystals."

\section{Acknowledgements}

I would like to thank Mr A. G. A. Cowie for performing a laparotomy on my patient, Dr D. S. Ridley for his histological report, and Dr R. R. M. Harman for supplying me with a case-history of his patient.

W. H. Jopling

\section{References}

Atkinson, A. J. Jr., Sheagren, J. N., Barba Rubio, J. and Knight, V. (1967). Evaluation of B 663 in human leprosy. Int. J. Lepr. 35, 119 ,

Browne, S. G. and Hogerzeil, L. M. (1962). B 663 in the treatment of leprosy. Preliminary report of a pilot trial. Lepr. Rev. 33, 6 .

Desikan, K. V., Ramanujam, K., Ramu, G. and Balakrishnan, S. (1975). Autopsy findings in a case of lepromatous leprosy treated with clofazimine. Lepr. Rev. 46, 181.

Harman, R. R. M. (1975). Pers. comm.

Helmy, H. S., Pearson, J. M. H. and Waters, M. F. R. (1971). Treatment of moderately severe ery thema nodosum leprosum with clof azimine-a controlled trial. Lepr. Rev. 42, 167.

Inkamp, F. M. J. H. (1968). A treatment of corticosteroid-dependent lepromatous patients in persistent erythema nodosum leprosum: a clinical evaluation of G.30320 (B663). Lepr. Rev. 39, 119.

Karat, A. B. A. (1975). Long-term follow-up of clofazimine (Lamprene) in the management of reactive phases of leprosy. Lepr. Rev. 46 (Suppl.) 105.

Pettit, J. H. S., Rees, R. J. W. and Ridley, D. S. (1967). Pilot trial of a riminophenazine derivative, B 663, in the treatment of lepromatous leprosy. Int. J. Lepr. 35, 25.

Schulz, E. J. (1971). Forty-four months' experience in the treatment of leprosy with clof azimine (Lamprene-Geigy). Lepr. Rev. 42, 178.

Williams, T. W. Jr., Mott, P. D., Wertlake, P. T., Barba Rubio, J., Adler, R. C., Hill, G. J., Perez Suarez, G. and Knight, V. (1965). Leprosy research at the National Institute of Health: experience with B 663 in the treatment of leprosy. Int. J. Lepr. 33, 767. 